\title{
Cross-Technology Localization: Leveraging Commodity WiFi to Localize Non-WiFi Device
}

\author{
Dian Zhang ${ }^{1 *}$, Rujun Zhang ${ }^{2}$, Haizhou Guo ${ }^{2}$, Peng Xiang ${ }^{2}$, Xiaonan Guo ${ }^{3}$ \\ ${ }^{1}$ Department of Computing and Decision Sciences, Lingnan University \\ 8 castle Peak Road, TuenMun, New Territories, Hong Kong \\ [e-mail: serena.dian@gmail.com] \\ ${ }^{2}$ College of Computer Science and Software Engineering, Shenzhen University, Shenzhen 518060, China \\ [e-mail: 2455308698@qq.com, 1800271045@email.szu.edu.cn, xiangpeng90812@gmail.com] \\ ${ }^{3}$ Department of Computer Science, Indiana University-Purdue University, Indianapolis, USA \\ [e-mail: xg6@iu.edu] \\ *Corresponding author: Dian Zhang
}

Received June 16, 2020; revised October 7, 2020; accepted January 3, 2021; published November 30, 2021

\begin{abstract}
Radio Frequency (RF)-based indoor localization technologies play significant roles in various Internet of Things (IoT) services (e.g., location-based service). Most such technologies require that all the devices comply with a specified technology (e.g., WiFi, ZigBee, and Bluetooth). However, this requirement limits its application scenarios in today's IoT context where multiple devices complied with different standards coexist in a shared environment. To bridge the gap, in this paper, we propose a cross-technology localization approach, which is able to localize target nodes using a different type of devices. Specifically, the proposed framework reuses the existing $\mathrm{WiFi}$ infrastructure without introducing additional cost to localize Non-WiFi device (i.e., ZigBee). The key idea is to leverage the interference between devices that share the same operating frequency (e.g., 2.4GHz). Such interference exhibits unique patterns that depend on the target device's location, thus it can be leveraged for cross-technology localization. The proposed framework uses Principal Components Analysis (PCA) to extract salient features of the received WiFi signals, and leverages Dynamic Time Warping (DTW), Gradient Boosting Regression Tree (GBRT) to improve the robustness of our system. We conduct experiments in real scenario and investigate the impact of different factors. Experimental results show that the average localization accuracy of our prototype can reach $1.54 \mathrm{~m}$, which demonstrates a promising direction of building cross-technology technologies to fulfill the needs of modern IoT context.
\end{abstract}

Keywords: Cross-technology, Channel State Information, Indoor Localization

This research is supported in part by NSFC 61872247, Shenzhen Peacock Talent Grant 827-000175, Guangdong Prenational Project 2014GKXM054, Guangdong Natural Science Foundation 2016A030313036, 2017 Guangdong Undergraduate Teaching Quality and Teaching Reform Project 839-0000026812. 


\section{Introduction}

This with the widespread popularity of Location-based services (LBS), our daily life have been greatly improved in many aspects. Global Positioning System (GPS) is one of the most popular and mature localization technique which facilitate many LBS applications. However, LBS of indoor environments can barely benefit from GPS, because GPS signals will be significantly attenuated by the buildings, which yields a low localization accuracy. Therefore, indoor localization technologies have attracted more and more attention from industry to academia recently. Among those, radio frequency (RF)-based technologies (e.g., WiFI, ZigBee, Bluetooth) are promising due to their open access and low cost.

Most of RF-based indoor localization techniques assume all the devices involved use the same commutation standard. For example, WiFi-based technologies require the devices complying with 802.11 protocol. Similarly, ZigBee-based technologies need the devices complying with 802.15.4 protocol. Most cooperative network localization technologies will consider network synchronization. Win et al. [1] presented the performance of wireless networks which can be significantly improved via the use of cooperation. Shen et al. [2] quantified the effect of clock asynchronism between anchors and agents on localization accuracy for networks. Khan et al. [3] introduced a distributed and iterative algorithm to locate sensors with unknown locations. Conti et al. [4] introduced the notion of network experimentation and developed a methodology particularly suited for cooperative wireless networks. In addition, RF-based solutions usually require established wireless communication between transceivers so that sophisticated signal analysis can be applied to determine devices' location. These assumptions limit the application scope of traditional RF-based localization system. For example, it is hard to localize a single device without any other devices that comply the same standard nearby. Therefore, it is desire to design a cross-technology localization system which releases the aforementioned assumptions. There are a few work aiming to solve this problem. ZiFi [5] identified the existence of WiFi networks through analyzing the WiFi interference caused by ZigBee devices. Croce et al. [6] detected and identified cross-technology interference on off-the-shelf WiFi cards by monitoring the sequence of receiver errors. But localization requires further investigation. ZiLoc [7] localized surrounding APs by constructing a Radio Signal Strength(RSS) fingerprint model. However, it needs dedicated devices (i.e., USRPs) and can only achieve room level accuracy.

To overcome the above limitations, we leverage commodity devices to achieve localization among heterogeneous wireless standards (i.e., ZigBee and WiFi). Specifically, we comprehensively analyze the characteristics of wireless signals through experiments, and observe interference between devices that share similar operating frequency (e.g., 2.4GHz). For example, in the presence of both ZigBee nodes and WiFi devices, the ZigBee signal will have interference with the WiFi signal, resulting in Channel State Information (CSI) changes at the WiFi receiver. Such changes have the potential to be utilized for localization. Note that, in this paper, we mainly focus on WiFi and ZigBee technologies, and the proposed solution can be extended to other wireless standards without much effort.

To implement localization based on devices with heterogeneous wireless standards, we propose Cross-Technology system to address several following challenges. First, it is challenging to determine and extract salient CSI features of different locations. Second, it is challenging to design a comparison mechanism that describes the similarity between the collected features and features from pre-collected profiles. To address the first challenge, our system uses CSI-waveform shape as features, because CSI extracted from WiFi receiver 
exhibits unique waveform shapes when a ZigBee node at different locations and causes interference. Moreover, our system determines the salient features of CSI-waveform based on Principle Component Analysis (PCA) which reduces the dimension of CSI data and removes the noise by utilizing correlated variations in CSI streams of different subcarriers. To address the second challenge, our system leverages two methods in localization, Dynamic Time Warping (DTW) [8] and Gradient Boosting Regression Tree (GBRT) [35]. DTW is used to estimate the similarity between two CSI-waveform shapes, and GBRT is robust to outliers. We perform out experiments in real scenario and test the localization accuracy of our system in the indoor environment. Experimental results show that the average localization accuracy of our system can reach $1.54 m$.

The main contributions of this paper are as follows. First, we designed and implemented a cross-technology localization prototype based on commodity devices (i.e., WiFi and ZigBee device). Second, we systematically studied the interference among nodes with heterogeneous wireless standards, and utilize PCA, DTW, GBRT to address different challenges in our algorithm design. Third, the experiment at the venue demonstrates that our system can achieve around $1 \mathrm{~m}$ localization accuracy.

The rest of this paper is organized as follows. We review related work in Section II. Then, we describe our system overview and methodology in Section III and Section IV, respectively. In Section V, we show the details of our system implementation and performance evaluation. Finally, we summarize our work and discuss possible future directions in Section VI.

\section{Related Work}

\subsection{RF-based Indoor Localization Technology}

Among RF-based indoor localization technologies, Received Signal Strength Indicator (RSSI)-based [9][10] and CSI-based [11][12] are two typical approaches.

RSS-based. Huang et al. [9] built a model to realize accurate ranging between any two nodes based on RSSI. Mazuelas et al. [10] proposed to dynamically estimate the propagation models through using RSS measurements. Mahapatra et al. [13] proposed an algorithm to improve accuracy of position estimation based on RSSI. However, RSSI could be easily affected by the environments due to multipath effect [12]. Xue et al. [14] presented the accurate RSSI extraction algorithm for Wi-Fi indoor localization. Kimoto et al. [15] presented a fingerprinting sensor localization system that improves the localization accuracy, it's achieved by extracting channel diversity from the RSS of WiFi access points measured on multiple ZigBee channels through fingerprinting localization. Also, RSSI had been shown as a highly volatile metric for indoor localization [16].

CSI-based. PhaseFi [17] localizes target devices based on fingerprinting the CSI information. Chapre et al. [11] built a location CSI signature through using Multiple Input Multiple Output (MIMO) technology. Zhang et al. [18] proposed a fingerprint system with CSI using MIMO-OFDM techology. Song et al. [19] proposed a CSI fingerprint-based indoor localization algorithm and built a CSI-based propagation model to realize localization.

CSI values are employed for indoor localization to avoid the instability of RSS values [20]. The above work all require the target nodes to apply the same radio technologies. In the design of our cross-technology localization system, we choose CSI as the resource since it consists of multiple dimension data which provide more information about channel condition. 


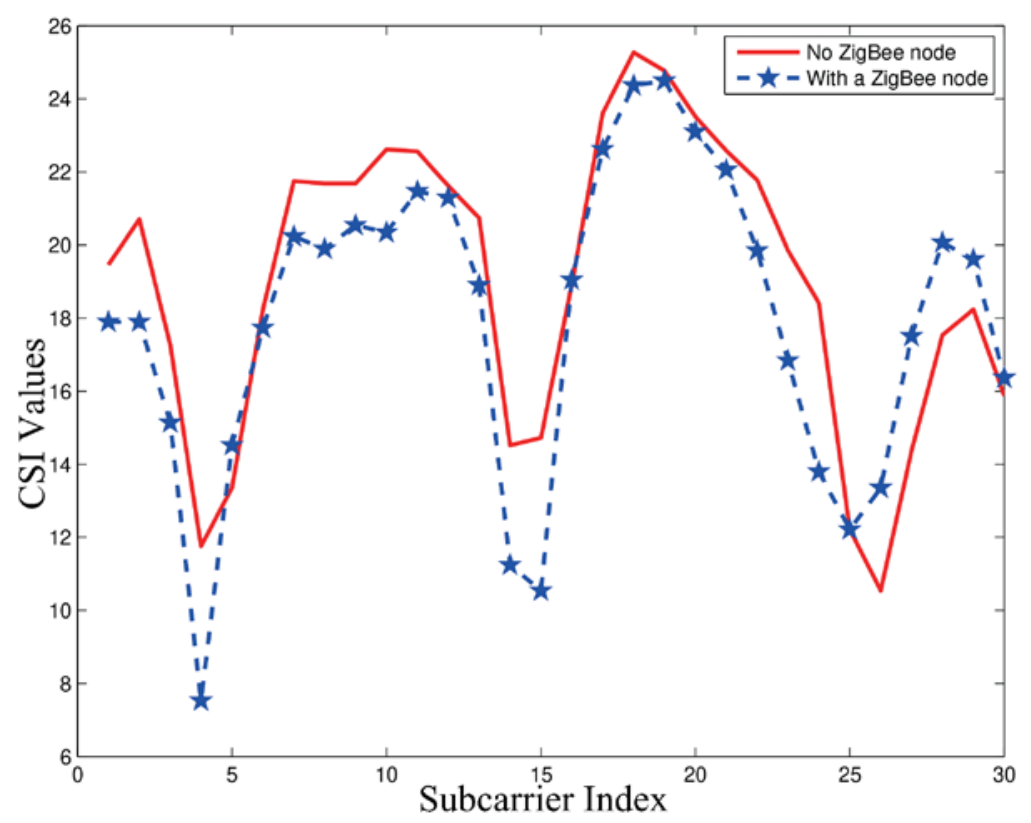

Fig. 1. Observation

\subsection{Cross-Technology}

Cross-technology had drawn many researchers' attentions in recent years [7][21][22][23]. They mainly aim to harmonize the coexistence and reduce the interference from other nodes with different technologies because coexistence often leads to low communication efficiency [21][24][25][26][27]. Qin [27] proposed a novel adaptive packet delivery(ADP) algorithm that enables ZigBee links to achieve enhanced performance under the presence of heavy WiFi traffic. Liu [22] designed a building fire monitoring system based on ZigBee-WiFi networks in which ZigBee nodes can transmit the information to WiFi network through a ZigBee-WiFi gateway. HoWiES [26] can save energy of WiFi nodes by utilizing ZigBee signal to wake up WiFi interface. A novel mechanism [23], referred as Cooperation Carrier Signaling (CCS), exploited to use one separate ZigBee node that emits a carrier signal concurrently with the desired ZigBee's data transmission. ZIMO [21] aimed to protect the ZigBee data packets so as to realize the coexistence of ZigBee and WiFi through the design of a cross-technology MIMO sink. A new approach [28] to support concurrent ZigBee transmissions over the same spectrum of a busy WiFi channel without incurring any extra delay or throughput loss to ZigBee. A novel ZigBee system [29] maintained high reliability even under saturated WiFi traffic, which is achieved by placing a ZigBee packet on the guard band of ongoing,ambient WiFi traffic to keep clear of interference from other WiFi. Chen et al. [30] proposed an accurate approach which utilizes per-byte SINR to detect corruption to improve the resilience of ZigBee transmissions. FreeBee [31] is able to realize direct unicast among WiFi, ZigBee, and Bluetooth through using side-channel.

There are also some work that able to detect the interference by other nodes in different technologies. ZiFi [5] only identified the existence of WiFi networks by using unique WiFi interference signature caused by ZigBee signals. Croce et al. [6] detected and identified cross-technology interference on off-the-shelf WiFi cards by monitoring the sequence of receiver errors and recognized the source of interference. But localization requires further investigated. ZiLoc [7] used ZigBee nodes to localize surrounding APs through building a Ra- 


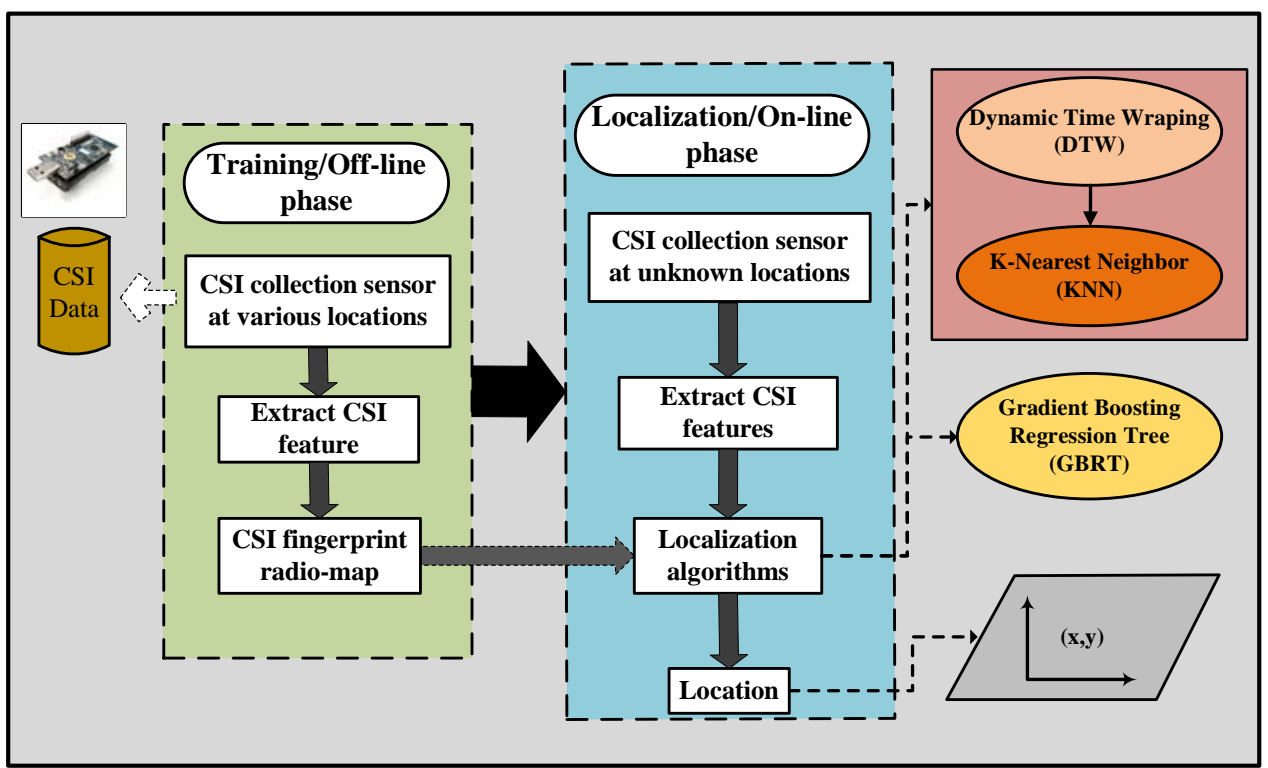

Fig. 2. System Architecture

dio Signal Strength (RSS) interference fingerprint model and computing the similarity between the online received RSS and the fingerprint. This work requires dedicated hardware (i.e., USRPs) and can only achieve room-level accuracy.

In contrast, our system achieves higher accuracy based on commodity devices. Moreover, it has the capability of localizing ZigBee devices by leveraging the communication interference between cross-technology (i.e., WiFi and ZigBee).

\section{System Overview}

\subsection{Channel State Information (CSI)}

Orthogonal Frequency Division Multiplexing (OFDM), as a bandwidth-efficient digital multicarrier modulation scheme, is widely used in IEEE $802.11 \mathrm{a} / \mathrm{g} / \mathrm{n}$. WiFi devices that support IEEE 802.11n standard always consist of multiple transmitting and multiple receiving antennas, which support Multiple-Input Multiple-Output (MIMO). These WiFi devices quantify the state of the channel in terms of CSI values. The CSI values essentially characterize the Channel Frequency Response (CFR) for each subcarrier between each Transmitter-Receiver (TX-RX) antenna pair.

Let $x_{i}$ and $y_{i}$ represent the transmitted and received signal vectors respectively. $N_{i}$ is the noise vector for the sub-carrier. $S_{\mathrm{c}}$ denote the number of OFDM sub-carriers. Then in narrow-band flat fading channel, a MIMO system is represented as:

$$
y_{i}=H_{i} x_{i}+N_{i}
$$

where $H_{i}$ represents the Channel State Information for the subcarrier $i$. For MIMO systems, $H_{i}$ is a $p * q$ matrix and is represented as: 
Table 1. Notations used in this paper

\begin{tabular}{|l|l|}
\hline Notation & Description \\
\hline$K$ & Number of CSI vectors for each location \\
\hline$N$ & Dimension of each target location \\
\hline$R_{i}^{K}, i \in[1, n]$ & $\begin{array}{l}\text { Input data(the CSI vectors during the online } \\
\text { phase) }\end{array}$ \\
\hline$T^{K}, i \in[1, n]$ & $\begin{array}{l}\text { Input data(the CSI vectors during the offline } \\
\text { phase) }\end{array}$ \\
\hline$y_{i}^{N}, i \in[1, n]$ & Output data(the target locations) \\
\hline$d i s t$ & The Euclidean distance \\
\hline$D_{i}$ & The distance of the $i$-th reference location to $T^{K}$ \\
\hline$w_{i}$ & The weight of the selected reference location $i$ \\
\hline$m$ & The number of the TX-RX antenna pairs \\
\hline$Q$ & The number of the basic regression trees \\
\hline$J$ & $\begin{array}{l}\text { The number of the leaf nodes of the regression } \\
\text { tree }\end{array}$ \\
\hline$h_{q}(R)$ & The $q$-th basic regression tree \\
\hline$C_{q j}$ & $\begin{array}{l}\text { The value for each leaf node of the regression } \\
\text { tree } h_{q}(R)\end{array}$ \\
\hline$f_{q}(R)$ & The integration of the basic regression trees \\
\hline$L$ & Cost function \\
\hline
\end{tabular}

$$
H_{i}=\left[\begin{array}{ccccc}
h_{11} & h_{12} & h_{13} & \cdots & h_{1 q} \\
h_{21} & h_{22} & h_{23} & \cdots & h_{2 q} \\
\cdots & \cdots & \cdots & \cdots & \cdots \\
h_{p 1} & h_{p 2} & h_{p 3} & \cdots & h_{p q}
\end{array}\right]
$$

where $p$ denotes the number of transmitting antennas, $q$ denotes the number of receiving antennas. Any two communicating WiFi devices estimate this channel matrix $H_{i}$ for every subcarrier by periodically transmitting a known packet of OFDM signals between each other. This leads to $S_{c}$ matrices with dimensions $p * q$ per CSI sample.

\subsection{Preliminary Study}

In this paper, we mainly aim to use WiFi signals to locate ZigBee nodes. Since WiFi and ZigBee share the same $2.4 \mathrm{GHz}$ ISM band, when they transmit wireless signals in the same environment, the ZigBee signals will interfere with the WiFi communication [32]. These interference can be captured in terms of CSI values for all subcarriers between every TX-RX antenna pair which can be further used to localize the ZigBee node. Fig. 1 shows the experiment result of our preliminary study, the solid line denotes that there is no ZigBee node in the wireless environment, whereas the star line denotes there is a ZigBee node. As the shown in Fig. 1, the ZigBee node in the environment has notable effects on the CSI values. 


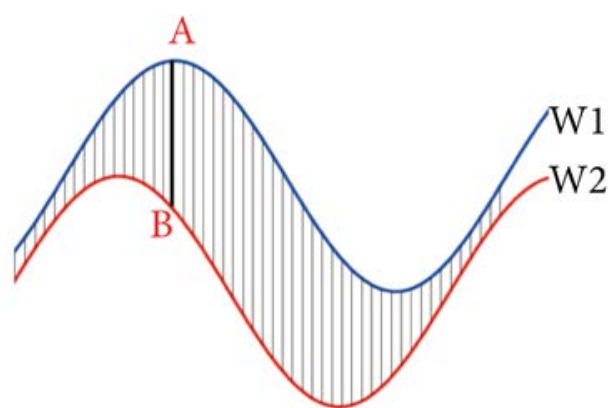

(a)

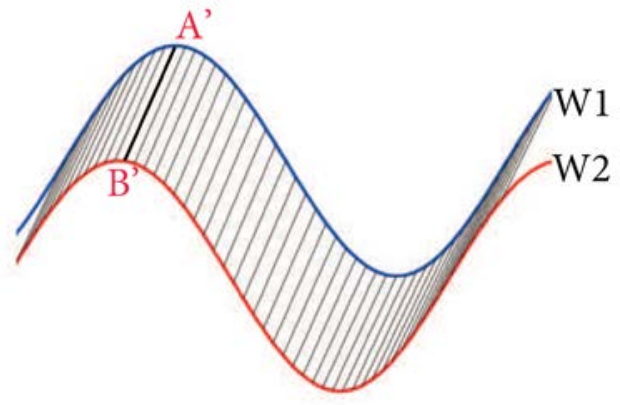

(b)

Fig. 3. W1 and W2 represent two waveform series. Linear alignment by the Euclidean distance does not produce satisfactory results, whereas the DTW distance adequately capture the similarity between the two waveform series due to the non-linear alignment. (a) Euclidean Distance; (b) DTW Distance

\subsection{System Architecture}

This section presents an explanation of our system architecture. As shown in Fig. 2, the working process of our system consists of two phases: training/off-line phase and localization/on-line phase. In the training/off-line phase, our system collects CSI data when a ZigBee node is at different locations. The insight is that the interference on the CSI is different when the target node is at different locations. Then, we extract features from the collected CSI data and further construct a CSI fingerprint radio-map. In the localization/on-line phase, our system continuously collects CSI data when ZigBee node appears and then extracts the features from CSI data, which is then compared with the features stored in the radio-map to estimate the location based on two algorithms including Dynamic Time Warping (DTW) [8][33] and Gradient Boosting Regression Tree (GBRT) [35].

\section{Methodology}

In this section, we present the algorithm design of our cross-technology localization system. Suppose there are $m$ TX-RX antenna pairs for the APs in the environments, which will act as both transmitter and receiver. The target object carries a ZigBee device which act as a transmitter and will interfere the WiFi signals. Our goal is to localize the ZigBee device. Some notations will be used in this paper are listed in Table 1.

\subsection{Data Collection}

Data collection can be divided into offline phase and online phase. During the offline phase, an AP continuously transmits wireless signals. A WiFi device receives the signals from a fixed position. The target ZigBee node was placed at each location to build a fingerprint radio-map which stored the CSI matrix $H$ for each pair of TX-RX antenna. We define the location space $R$ as a set of $n$ points on the ground. $R$ is denoted as $R=\left\{R_{1}, R_{2}, \ldots, R_{n}\right\}$. During the online phase, when the target ZigBee node appeared, it will also interfere the WiFi signals. We 
collected the CSI information and used our localization algorithms to localize the target position.

\subsection{Feature Extraction}

In our system, we utilize the Principal Component Analysis (PCA) [34] algorithm to extract the interference features. Specifically, this process contains the following three steps:

1) Preprocess CSI Data: Let $m$ denote the number of the TX-RX antenna pairs. Thus, for the CSI of the subcarrier $i$, we can reshape the $p * q$ dimensions as $m * 1$ dimensions: $H_{i}=[h(1), h(2), \ldots, h(m)]^{T}$. Then the received $S_{c}$ subcarriers can be expressed as: $H=\left[H_{1}, H_{2}, \ldots, H_{S_{c}}\right]$. We normalize the matrix $H$ so that each column has zero mean and unit variance, denoted as $Z$.

$$
Z=\left[Z_{1}, Z_{2}, \ldots, Z_{S_{c}}\right]=\left[\begin{array}{ccccc}
Z_{1}(1) & Z_{2}(1) & Z_{3}(1) & \cdots & Z_{S_{c}}(1) \\
Z_{1}(2) & Z_{2}(2) & Z_{3}(2) & \cdots & Z_{S_{c}}(2) \\
\cdots & \cdots & \cdots & \cdots & \cdots \\
Z_{1}(m) & Z_{2}(m) & Z_{3}(m) & \cdots & Z_{S_{c}}(m)
\end{array}\right]
$$

2) Calculate the Covariance Matrix and Eigenvalue Decomposition: Let Cov represent the covariance matrix of the matrix $Z$, then $\operatorname{Cov}=\frac{1}{m} Z^{T} * Z$. Since the covariance matrix is square, we can calculate the eigenvectors and eigenvalues for this matrix. Let $\Lambda$ denote the matrix whose each element is an eigenvalue $\lambda$ and these eigenvalues are sorted in descending order. Let $W$ denote the matrix whose each element is the eigenvector $w$ and it corresponds to each element of $\Lambda$. Then $\Lambda$ and $W$ can be represented as:

$$
\begin{aligned}
& \Lambda=\left[\begin{array}{lllll}
\lambda_{1} & & & & \\
& \lambda_{2} & & & \\
& & \lambda_{3} & & \\
& & & \ldots & \\
& & & & \lambda_{S_{c}}
\end{array}\right] \\
& W=\left[w_{1}, w_{2}, \ldots, w_{S_{c}}\right]
\end{aligned}
$$

We select $d$ corresponding eigenvectors to form the compression matrix $U$. The choice of $d$ is the minimum of the following conditions:

$$
\frac{\sum_{i=1}^{d} \lambda_{i}}{\sum_{i=1}^{S_{c}} \lambda_{i}} \geq t
$$




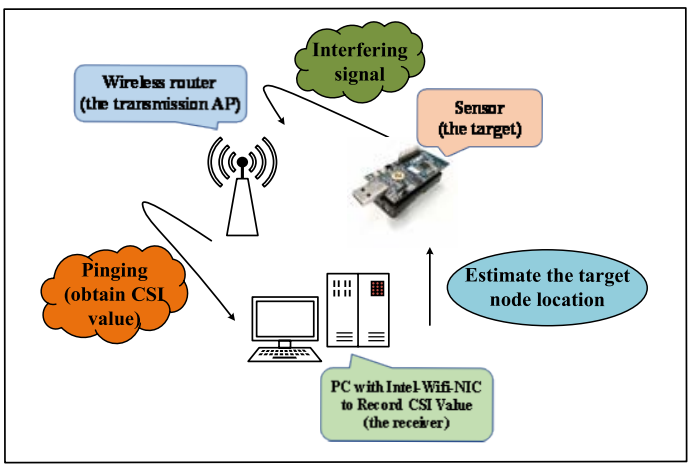

(a)

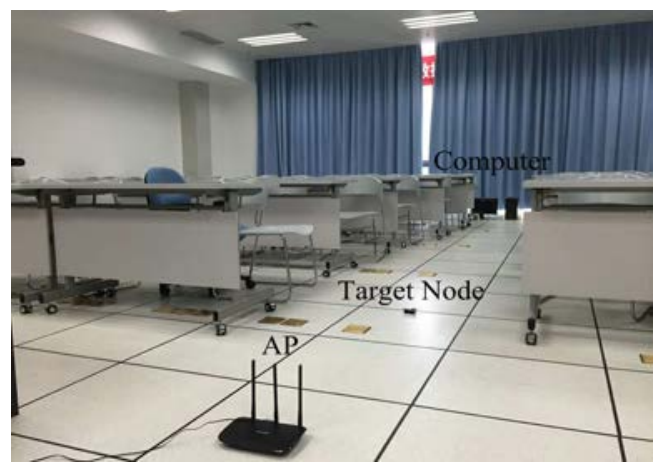

(b)

Fig. 4. System and Experiment Scenario. (a) System; (b) Classroom

$$
U=\left[w_{1}, w_{2}, \ldots, w_{d}\right]
$$

where $t$ is the cumulative contribution rate.

3) Derive the Principal Component and Build the Fingerprint Radio-map: The CSI values after dimension reduction can be expressed as $H^{*}=H^{*} U, H^{*}$ is a $m * d$ matrix. Hence, we can generate a radio-map that stores the fingerprints of all the locations for a given floor-plan.

\subsection{Localization Algorithm}

\subsubsection{DTW Algorithm}

To estimate the location of a ZigBee node, we use the DTW algorithm to calculate the distance between the received CSI vectors and the training vectors stored in the fingerprint Radio-map. Then, we use the KNN algorithm to estimate the node location.

1) Preprocess CSI Data: Since the feature extracted from CSI vectors is a waveform that has non-linear alignment and thus cannot be compared using standard method like correlation coefficient or Euclidean distance. The DTW distance adequately captures the similarity between the two waveform series due to the non-linear alignment [33]. Therefore, we utilize the DTW algorithm to calculate the distance. Fig. 3 shows the difference between the Euclidean distance and the DTW distance for the waveform having distortion on the time axis.

From the previous subsection, we have $K=m * d$ number of CSI vectors for each location. And when the target node appears, we get the CSI vector $T^{K}$. By using DTW, we can calculate the vector distance between $T^{K}$ and each $R_{i}^{K}(1 \leq i \leq n)$. The first stage of the DTW algorithm is to fill a local distance matrix which we denote as dist. This matrix represents the Euclidean distance between every two points in the waveform series:

$$
\operatorname{dist}(j, k)=\left|R_{i}^{j}-T^{k}\right|
$$




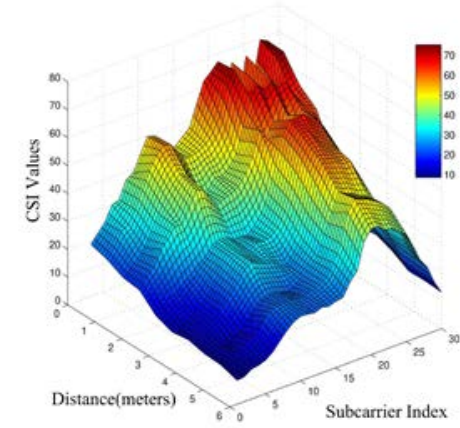

(a)

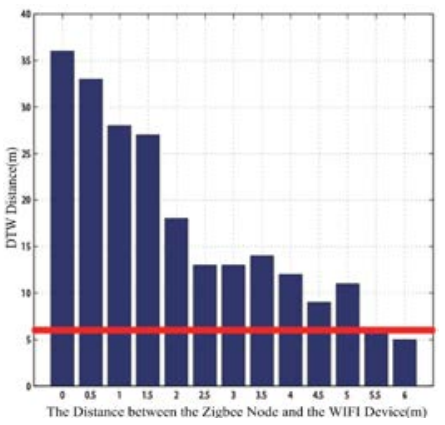

(b)

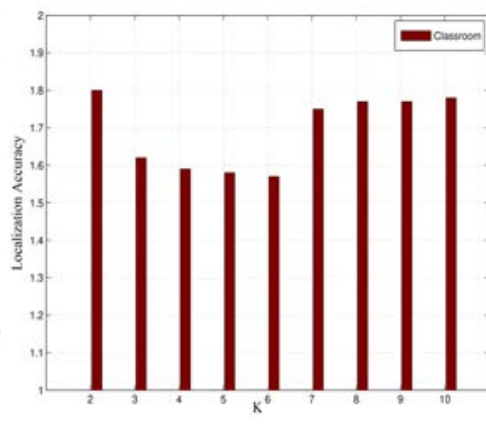

(c)

Fig. 5. The Impact of Parameters. (a) The Impact of Distance on CSI Values; (b) The Impact of Distance on CSI Values(The red horizontal line represents the DTW distance of the CSI values between two different moments without the presence of ZigBee node); (c) The Impact of K Values

where $j$ is the $j$-th CSI value of $R_{i}$, and $k$ is the $k$-th CSI value of $T$. In the second stage, it fills the warping matrix $D_{i}$. The following equation describes the initial condition of the algorithm.

$$
D_{i}\left(R_{i}^{j}, T^{k}\right)= \begin{cases}\infty, & \text { if } j=0 \text { and } k=0 \\ 0, & \text { if } j=k=0\end{cases}
$$

Then, we use the following equation to calculate the distance between $T^{K}$ and each $R_{i}^{K}$, when $j$ is larger than 1 or $k$ is larger than 1 .

$$
D_{i}\left(R_{i}^{j}, T^{k}\right)=\operatorname{dist}(j, k)+\min \left\{\begin{array}{c}
D_{i}\left(R_{i}^{j-1}, T^{k}\right) \\
D_{i}\left(R_{i}^{j}, T^{k-1}\right) \\
D_{i}\left(R_{i}^{j-1}, T^{k-1}\right)
\end{array}\right.
$$

For total $n$ number of the reference locations, we define a set $D$ to store the distance of the $n$ locations to $T^{K}$ calculated by the above equation.

$$
\begin{gathered}
D=\left\{D_{1}, D_{2}, \ldots, D_{n}\right\} \\
D_{i}=D_{i}\left(R_{i}^{j}, T^{k}\right), 1 \leq i \leq n
\end{gathered}
$$

2) Estimate the Target Node Location: We utilize the KNN algorithm to estimate the target node's location. After obtaining the DTW distance set between $n$ reference locations to the target node, we choose $k$ reference locations with the smallest DTW distance as the nearest neighboring reference locations. The target node's coordinate $\left(y^{1}, y^{2}\right)$ is calculated by 


$$
\left(y^{1}, y^{2}\right)=\sum_{i=1}^{k} w_{i}\left(y_{i}^{1}, y_{i}^{2}\right), w_{i}=\frac{1 / D_{i}^{2}}{\sum_{i=1}^{k} 1 / D_{i}^{2}}
$$

where $\left(y_{i}^{1}, y_{i}^{2}\right)$ denotes the coordinate of the selected reference location $i . w_{i}$ is the weight of the selected reference location $i$. The choice of $k$ determines the accuracy of localization.

\subsubsection{GBRT Algorithm}

When we use the GBRT algorithm to estimate the node location, for the CSI of each location, we reshape the $m * d$ dimensions as $1 * K$ dimensions by rows: $R_{i}=\left[R_{i}(1), R_{i}(2), \ldots, R_{i}(K)\right], R_{i} \in \mathfrak{R}^{K}$. Let $y_{i}^{N}$ denote each location on the ground, $y_{i}^{N} \in \mathfrak{R}^{N}$, where $N$ is dimension of the node location on the ground. Here, its value is 2 . $\left\{y_{i}^{2}\right\}_{i=1}^{n}$ represents the target node's coordinates $\left\{\left(y_{i}^{1}, y_{i}^{2}\right)\right\}_{i=1}^{n}$ on the ground. Given the training set $\left\{\left(R_{i}, y_{i}^{2}\right)\right\}_{i=1}^{n}$ with $n$ numbers of input variables $R_{i}$ and output variables $y_{i}^{2}$. Our goal is to find basic regression trees $h(R)$ that minimizes the expected value of the loss function $L\left(y^{2}, f(R)\right)$ to build the optimal GBRT model. GBRT needs to be initialized with a constant $c$ and then builds the model. The GBRT model is formulated as follows:

$$
\begin{gathered}
f_{0}(R)=\arg \min _{c} \sum_{i=1}^{n} L\left(y_{i}^{2}, c\right) \\
f_{q}(R)=f_{q-1}(R)+\arg \min _{\mathfrak{R}_{q j}} \sum_{i=1}^{n} L\left(y_{i}^{2}, f_{q-1}\left(R_{i}\right)+h_{q}\left(R_{i}\right)\right)
\end{gathered}
$$

where $f_{0}(R)$ is a constant function. $h_{q}(R)$ is the $q$-th basic regression tree. $\Re_{q j}$ is the leaf node region of the $q$-th regression tree. $f_{q}(R)$ is the integration of the basic regression trees.

For GBRT, the tree model is optimized by the gradient descent method. For $q=1,2, \ldots, Q$, $i=1,2, \ldots, n$, the negative gradient $r_{q j}\left(R_{i}\right)$ is calculated by

$$
r_{q j}\left(R_{i}\right)=-\left[\frac{\partial L\left(y_{i}^{2}, f\left(R_{i}\right)\right)}{\partial f\left(R_{i}\right)}\right]_{f(R)=f_{q-1}(R)}
$$

We are learning a regression tree $h_{q}(R)$ given a new training data : $D=\left\{\left(R_{1}, r_{q 1}\right), \ldots,\left(R_{n}, r_{q n}\right)\right\}$. Then we can minimize the residual by choosing proper $c_{q j}$ for each leaf node of the regression tree, the value is $c$. For $j=1,2, \ldots, J$, we can calculate $c_{q j}$ by: 


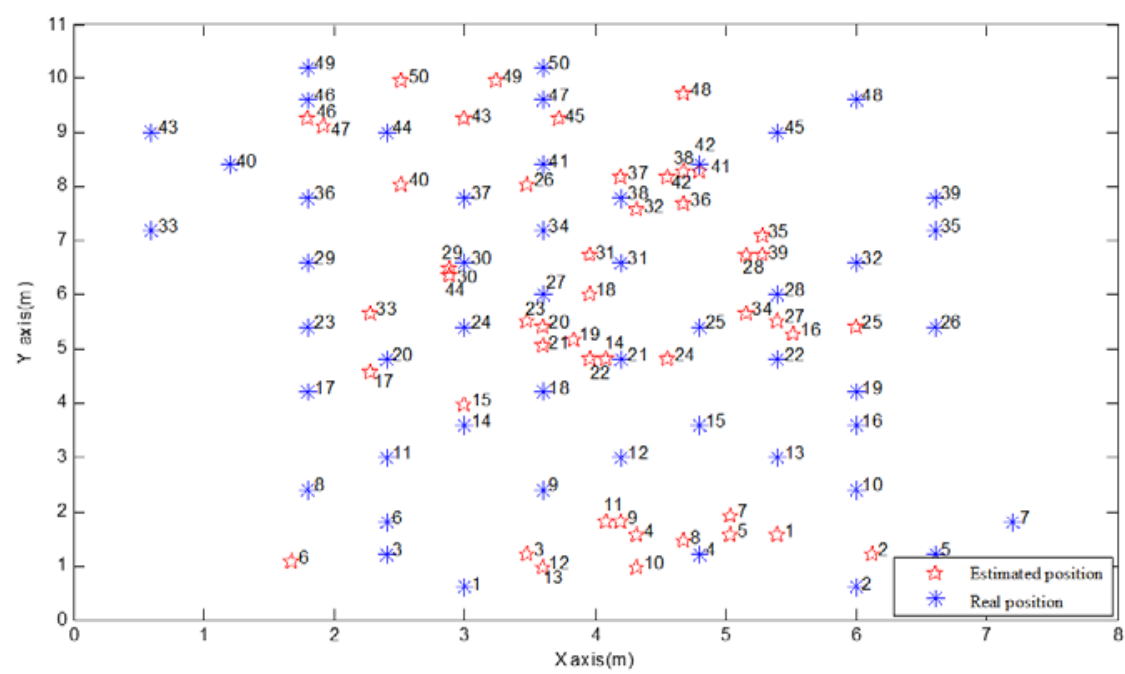

Fig. 6. Localization Result with DTW

$$
c_{q j}=\arg \min _{c} \sum_{R_{i} \in \Re_{q j}} L\left(y_{i}^{2}, f_{q-1}\left(R_{i}\right)+c\right)
$$

The tree function can be updated as:

$$
f_{q}(R)=f_{q-1}(R)+\sum_{j=1}^{J} c_{q j} I\left(R \in \mathfrak{R}_{q j}\right)
$$

The final regression tree model can be represented as:

$$
f_{Q}(R)=\sum_{q=1}^{Q} \sum_{j=1}^{J} c_{q j} I\left(R \in \mathfrak{R}_{q j}\right)
$$

The accuracy of localization is affected by the number of basic regression trees, learning rate and max depth of the regression tree. $Q$ is the number of basic regression trees for the GBRT model. $J$ represents the learning rate. Let $l$ represent the max depth of the regression tree.

The upper method is included in the Python-based library SCIKIT-LEARN. We utilize it to train a GBRT model $f_{Q}(R)$ by the training set $\left\{\left(R_{i}, y_{i}^{2}\right)\right\}_{i=1}^{n}$. After we get this model, when a new CSI vector is received in a location, we can predict the target location by using this GBRT model. 


\section{Implementation and Evaluation}

\subsection{Experimental Setup}

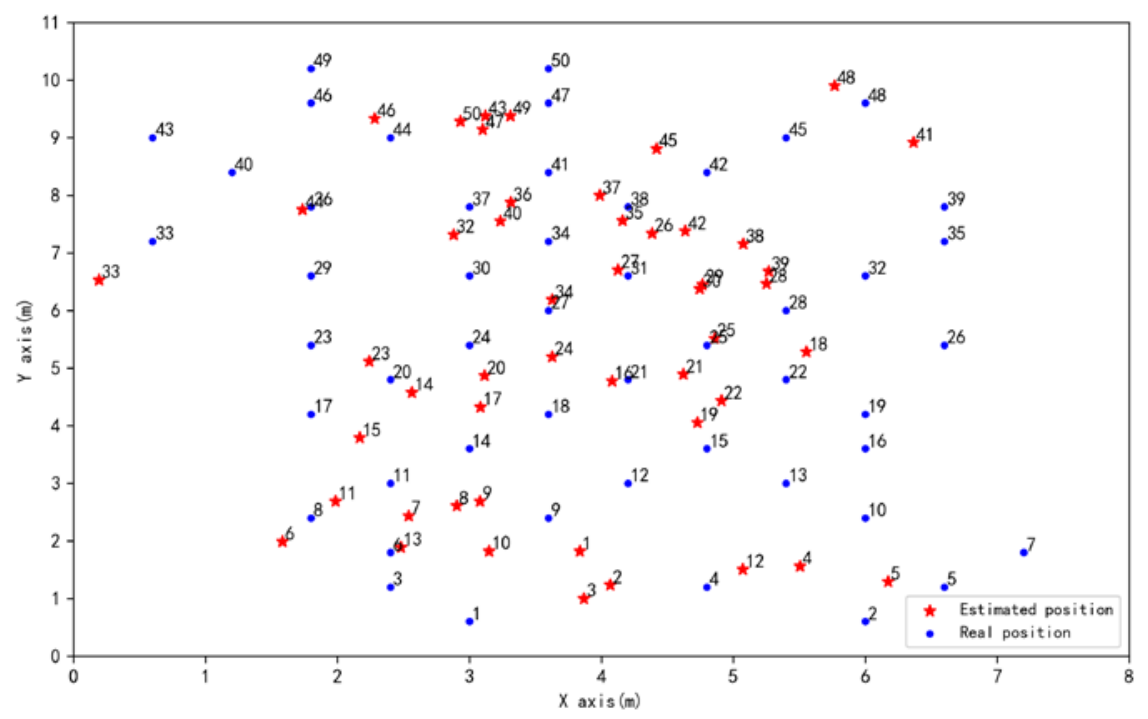

Fig. 7. Localization Result with GBRT

As shown in Fig. 4(a), our system consists of one TP-Link TL-WR880N WiFi router as the transmission AP, a Lenovo desktop computer as a receiver and one ZigBee node as the target. The AP and the desktop computer are stationary. The Lenovo desktop is equipped with an Intel WiFi Link 5300 NIC with three antennas. In our experiment, we set the target node's broadcast interval as 0.03 s to transmit a packet. The CSI values between WiFi Nodes are obtained from ICMP packets by pinging the WiFi device, which is then processed by MATLAB in real-time. For each TX-RX antenna pair, we get $S_{c}=30$ OFDM subcarriers of $20 \mathrm{MHz}$ WiFi Channel. In the offline phase of our system, GBRT parameters $Q, J$ and $l$, are set as 200, 0.1 and 3 , respectively by default.

\subsection{The Effective Range Between ZigBee Node and WiFi Device}

In this subsection, we investigate the effective range between ZigBee node and WiFi device. After performing many experiments, we observed that the CSI values vary with distances, specifically, the CSI values increase when the node moves closer to the wireless router as shown in Fig. 5(a).

Since the ZigBee is a low powered device with short transmission range. When the distance between the ZigBee node and the WiFi device exceeds a certain range, the interference becomes negligible and hence cannot locate the ZigBee node. In order to test the effective range, we choose $0 m$ to $6 m$ with an interval of $0.5 m$ as shown in Fig. 5(b). The x-axis represents the distance between the ZigBee node and the WiFi device. Similarly, the y-axis represents the DTW distance of the CSI values between the waveform with and without ZigBee. The red horizontal line represents the DTW distance of the CSI values between two different moments without the presence of ZigBee node, which is the threshold to decide 
whether a ZigBee node exists or not. We find that, when the distance between the ZigBee node and the WiFi device is more than $5 \mathrm{~m}$, the DTW distance is below the horizontal line. In this experiment, since we place WiFi devices at the center, the effective range can reach $5 \mathrm{~m}$ radius.

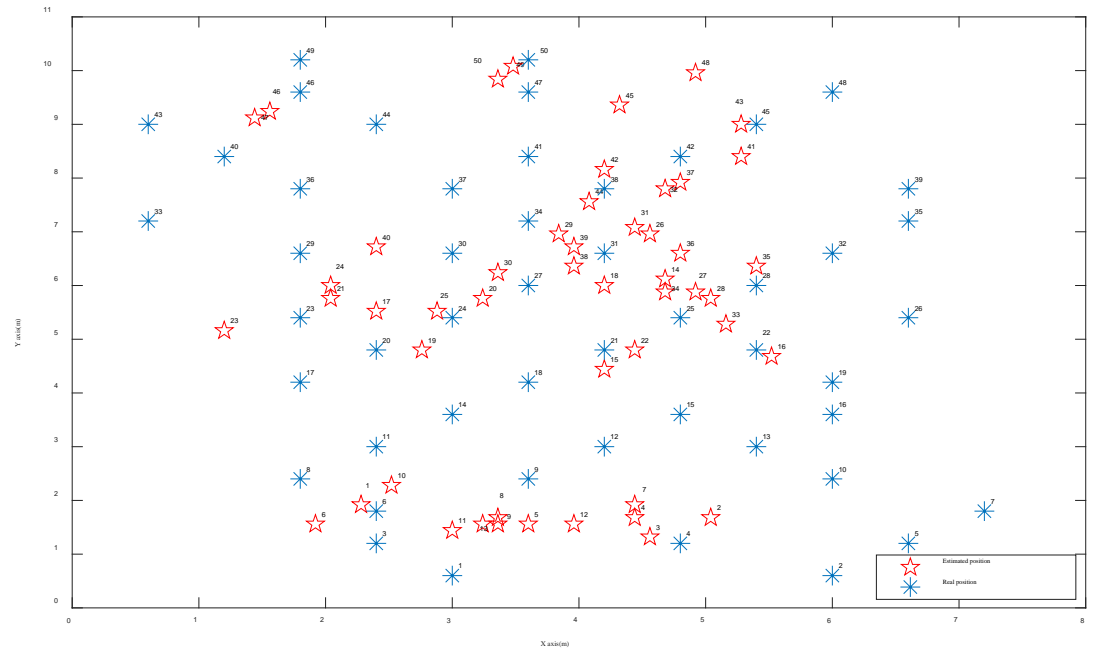

Fig. 8. Localization Result with KNN

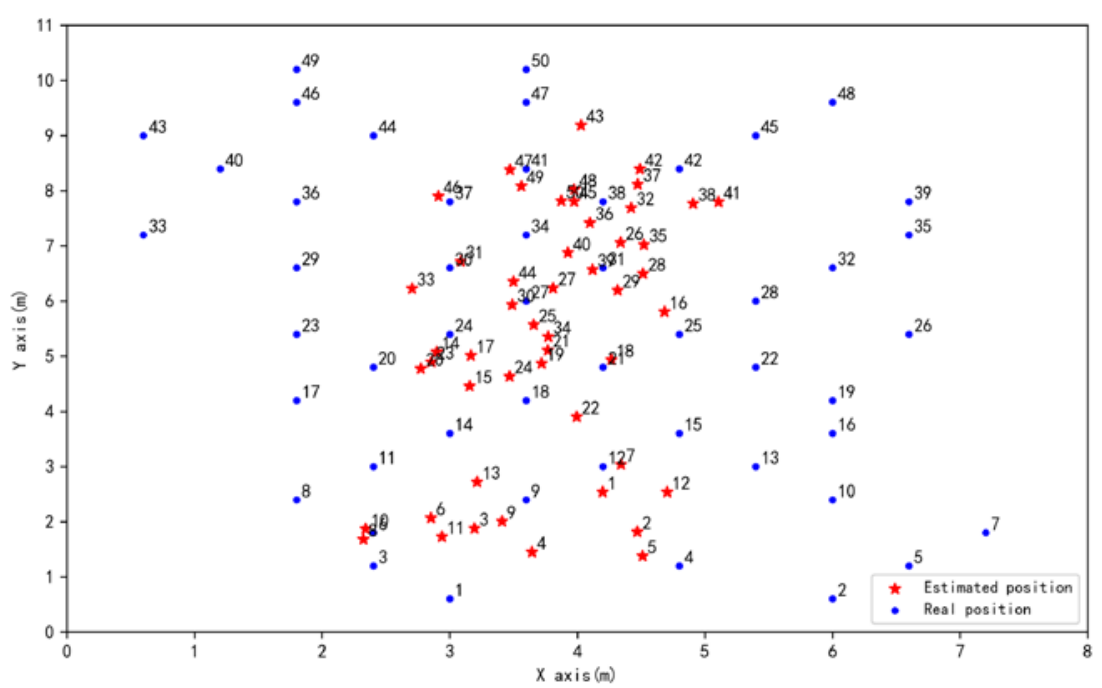

Fig. 9. Localization Result with SVR

\subsection{Impact of $\boldsymbol{k}$ in DTW Algorithm}

In this subsection, we investigate the impact of parameter $k$ on KNN. The $k$ represents number of neighboring reference locations that influence the estimate of localization accuracy. $k$ is set from 2 to 10 during the experiment. We select a scenario to conduct this experiment. The scenario is a $9 m \times 12 m$ classroom, which is shown in Fig. 4(b). We divide the floor of the classroom into different grids as the possible target locations. We randomly select 50 locations 
to test in the classroom. We compute the average localization accuracy. The experimental results are as shown in Fig. 5(c), we observe that the localization accuracy varies with the value of $k$, but the error is minimal when $k=6$.

\subsection{Localization Accuracy}

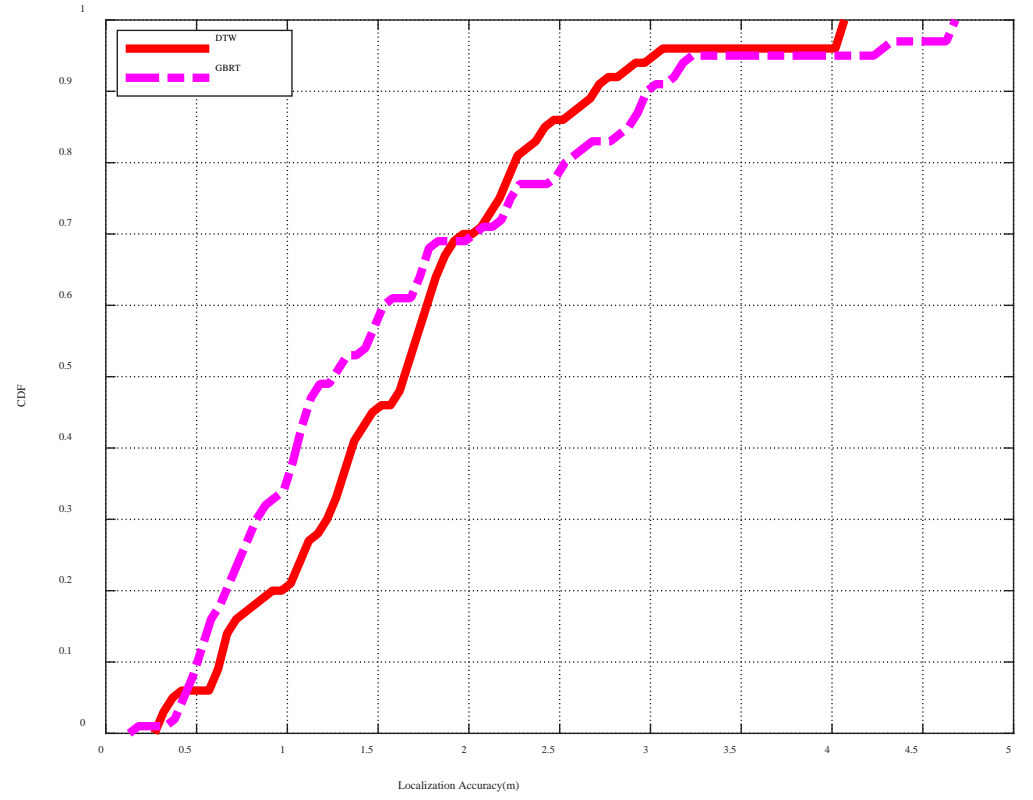

Fig. 10. Comparison of Different Algorithms in Cross-Technology System

In this subsection, we will show the performance of our algorithms. There are a lot of tables in the classroom, which is a typical multipath environment. Fig. 6 and Fig. 7 show the result of localization with DTW and GBRT respectively, the blue stars with numbers indicate the real locations, and the red five-pointed stars indicate the estimated position. Fig. 6 and Fig. 7 show localization in classroom. We randomly tested 50 locations in classroom. As shown in Fig. 6 and Fig. 7, localization accuracy is worse when the target is near the walls compared to the center part. There are two possible reasons. This first reason is the points near the walls have more interference from the wall. The second reason is that the WiFi devices are placed in the center part experimental area. Our experiment shows that the average localization accuracy with DTW and GBRT in classroom are $1.58 \mathrm{~m}$ and $1.54 \mathrm{~m}$, respectively.

\subsection{Algorithm Comparison}

In this subsection, we compare the algorithms in our Cross-Technology system first. We select the classroom to compare the DTW and GBRT algorithm. Fig. 10 shows the CDF of DTW and GBRT algorithms. The average localization accuracy with GBRT is better than DTW, but DTW is easier to implement and need not be trained to get the parameter model.

In this paper, the popular KNN and Support Vector Regression (SVR) algorithm are used as the benchmarks. We compare our algorithms with KNN and SVR. For KNN, the localization error is minimal when $k=6$. For SVR, we utilized the grid search method to select the optimal parameters penalty factor $c$, gamma and kernel function, where $c$ is the penalty factor which represents the tolerance of the error, $\gamma$ is the parameter which comes from kernel 
function. So the optimal parameter combination shows that the kernel function is Radio Basis Function (RBF), the value of $c$ is 1 and the value of $\gamma$ is 0.1 . We select the classroom to compare the performance of our algorithms with KNN and SVR, which is shown in Fig. 6, Fig. 7, Fig. 8 and Fig. 9, respectively. Fig. 11 shows the CDF of DTW, GBRT, SVR and KNN results. Our experiment shows that the average localization accuracy of DTW, GBRT, KNN and SVR in classroom are $1.58 \mathrm{~m}, 1.54 \mathrm{~m}, 1.78 \mathrm{~m}$ and $1.72 \mathrm{~m}$, respectively. As shown in Fig. 8 and Fig. 9, our algorithm outperforms KNN and SVR by $13 \%$ and 10\%, respectively.

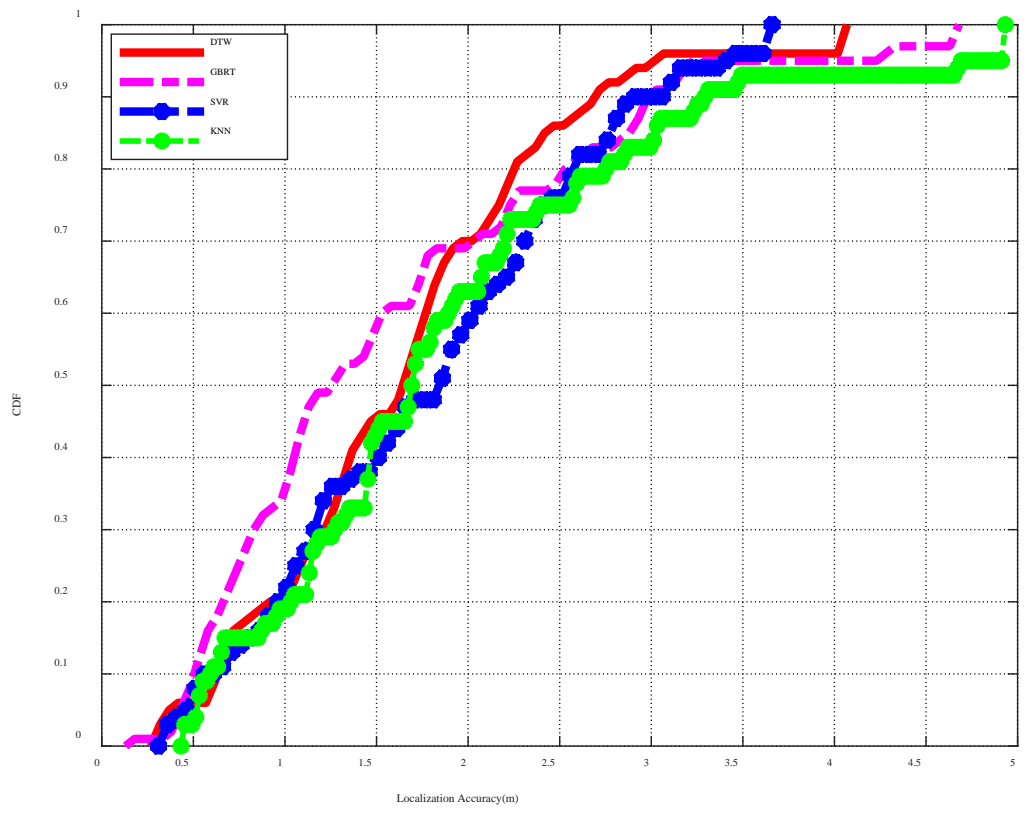

Fig. 11. Comparison of Different Algorithms

\subsection{Latency}

In our system, there are two main phases. 1) Training phase to build fingerprint radio-map. 2) Testing phase to estimate the target ZigBee node location. Only the testing phase influences latency. In our system, the wireless router needs about $0.8 \mathrm{~ms}$ to transmit a packet with 100 bytes beacon message in IEEE 802.11n. The other computation time can be neglected. When the target node appears at any given position, we collect 100 CSI data samples and the latency will be around $100 \times 0.8=80 \mathrm{~ms}$.

\subsection{Discussion}

The assumption of this paper is that the interference node should use the same channel with the WiFi node. Considering the $2.4 \mathrm{GHz}$, operating frequency is very popular in many mobile devices, it is feasible in real use. Another limitation is that the radio environment is relatively clean. It is our first try. Later we will utilize the interference pattern to distinguish different wireless nodes with different protocols. 


\section{Conclusion and Future Work}

In this paper, we design and implement a Cross-Technology localization system. In our prototype, we localize ZigBee device by leveraging its interference on the WiFi signal. CSI collected at the receiver can characterize this interference by detecting the changes of the wireless channel frequency response. Specifically, we utilize PCA algorithm to extract main features of the interference, use DTW and GBRT algorithm to calculate the location of the target. We implement our system using a Commercial Off-The-Shelf (COTS) WiFi router and a Lenovo desktop, and evaluated the system in real environment. Our experimental results show that our system can achieve localization accuracy of $1.54 \mathrm{~m}$, which dramatically outperforms the room-level accuracy of traditional technologies. In the future, we plan to implement our system in GUN Radio-USRP platform, since it can obtain more subcarrier information compared with ours. In addition, we will conduct more experiments in a larger place to measure the scalability of our system.

\section{References}

[1] M. Z. Win, A. Conti, S. Mazuelas, Y. Shen, W. M. Gifford, D. Dardari, and M. Chiani, "Network localization and navigation via cooperation,” IEEE Commun. Mag, vol. 49, no. 5, pp. 56-62, May. 2011. Article (CrossRef Link)

[2] Y. Shen and M. Z. Win, "Fundamental limits of wideband localization - Part I: A general framework,” IEEE Trans. Inf. Theory, vol. 56, no. 10, pp. 4956-4980, Oct. 2010. Article (CrossRef Link)

[3] U. A. Khan, S. Kar, and J. M. F. Moura, "Distributed sensor localization in random environments using minimal number of anchor nodes,” IEEE Trans. Signal Process, vol. 57, no. 5, pp. 2000-2016, May. 2009. Article (CrossRef Link)

[4] A. Conti, M. Guerra, D. Dardari, N. Decarli, and M. Z. Win, "Network experimentation for cooperative localization,” IEEE J. Sel. Areas Commun, vol. 30, no. 2, pp. 467-475, Feb. 2012. Article (CrossRef Link)

[5] D. Croce, D. Garlisi, F. Giuliano, N. Inzerillo, and I. Tinnirello, "Learning from errors: Detecting cross-technology interference in wifi networks," IEEE Transactions on Cognitive Communications and Networking, vol. 4, no. 2, pp. 347-356, 2018. Article (CrossRef Link)

[6] J. Niu, B. Lu, L. Cheng, Y. Gu, and L. Shu, "Ziloc: Energy efficient wifi fingerprint-based localization with low-power radio," in Proc. of 2013 IEEE Wireless Communications and Networking Conference, pp. 4558-4563, April. 2013. Article (CrossRef Link)

[7] J. Wang and D. Katabi, "Dude, where's my card?: Rfid positioning that works with multipath and non-line of sight,” in Proc. of SIGCOMM, pp. 51-62, 2013. Article (CrossRef Link)

[8] J. Yu, J. Amores, N. Sebe, P. Radeva, and Q. Tian, "Distance learning for similarity estimation," IEEE Transactions on Pattern Analysis and Machine Intelligence, vol. 30, no. 3, pp. 451-462, March. 2008. Article (CrossRef Link)

[9] M. Shin and I. Joe, "An indoor localization system considering channel interference and the reliability of the rssi measurement to enhance location accuracy," in Proc. of 2015 17th International Conference on Advanced Communication Technology, pp. 583-592, July. 2015. Article (CrossRef Link)

[10] S. Mazuelas, A. Bahillo, R. M. Lorenzo, P. Fernandez, F. A. Lago, E. Garcia, J. Blas, and E. J. Abril, "Robust indoor positioning provided by real-time rssi values in unmodified wlan networks," IEEE Journal of Selected Topics in Signal Processing, vol. 3, no. 5, pp. 821-831, Oct. 2009. Article (CrossRef Link)

[11] Y. Chapre, A. Ignjatovic, A. Seneviratne, and S. Jha, "Csi-mimo: Indoor wi-fi fingerprinting system," in Proc. of 39th Annual IEEE Conference on Local Computer Networks, pp. 202-209, 2014. Article (CrossRef Link) 
[12] K. Wu, J. Xiao, Y. Yi, D. Chen, X. Luo, and L. M. Ni, “Csi-based indoor localization,” IEEE Transactions on Parallel and Distributed Systems, vol. 24, no. 7, pp. 1300-1309, July. 2013.

[13] R. K. Mahapatra and N. S. V. Shet, "Localization based on rssi exploiting gaussian and averaging filter in wireless sensor network," Arabian Journal for Science and Engineering, vol. 43, no. 8, pp. 4145-4159, 2018. Article (CrossRef Link)

[14] W. Xue, W. Qiu, X. Hua, and K. Yu, "Improved wi-fi rssi measurement for indoor localization," IEEE Sensors Journal, vol. 17, no. 7, pp. 2224-2230, 2017. Article (CrossRef Link)

[15] R. Kimoto, S. Ishida, T. Yamamoto, S. Tagashira, and A. Fukuda, "Muchloc: Indoor zigbee localization system utilizing inter-channel characteristics,” Sensors, vol. 19, no. 7, pp. 1645, 2019. Article (CrossRef Link)

[16] Y. Chapre, P. Mohapatra, S. Jha, and A. Seneviratne, "Received signal strength indicator and its analysis in a typical wlan system (short paper)," in Proc. of 38th Annual IEEE Conference on Local Computer Networks, pp. 304-307, Oct. 2013. Article (CrossRef Link)

[17] X. Wang, L. Gao, and S. Mao, "Csi phase fingerprinting for indoor localization with a deep learning approach,” IEEE Internet of Things Journal, vol. 3, no. 6, pp. 1113-1123, Dec. 2016. Article (CrossRef Link)

[18] L. Zhang, E. Ding, Z. Zhao, Y. Hu, X. Wang, and K. Zhang, "A novel fingerprinting using channel state information with mimocofdm,” Cluster Computing, vol. 20, no. 4, pp. 3299-3312, 2017. Article (CrossRef Link)

[19] Q. Song, S. Guo, X. Liu, and Y. Yang, "Csi amplitude fingerprinting based nb-iot indoor localization,” IEEE Internet of Things Journal, vol. 5, no. 3, pp. 1494-1504, 2018. Article (CrossRef Link)

[20] Z. Yang, Z. Zhou, and Y. Liu, "From rssi to csi: Indoor localization via channel response," ACM Computing Surveys, vol. 46, no. 2, pp. 1-32, 2013. Article (CrossRef Link)

[21] Y. Yubo, Y. Panlong, L. Xiangyang, T. Yue, Z. Lan, and Y. Lizhao, "ZIMO: building cross-technology MIMO to harmonize zigbee smog with WiFi flash without intervention," in Proc. of the 19th annual international conference on Mobile computing and networking, pp. 465-476, 2013. Article (CrossRef Link)

[22] L. Yunhong and Q. Meini, "The design of building fire monitoring system based on zigbee-wifi networks," in Proc. of 2016 Eighth International Conference on Measuring Technology and Mechatronics Automation, pp. 733-735, March. 2016. Article (CrossRef Link)

[23] X. Zhang and K. G. Shin, "Cooperative carrier signaling: Harmonizing coexisting wpan and wlan devices,” IEEE/ACM Transactions on Networking, vol. 21, no. 2, pp. 426-439, April. 2013. Article (CrossRef Link)

[24] S. Rayanchu, A. Patro, and S. Banerjee, "Airshark: Detecting non-wifi rf devices using commodity wifi hardware," in Proc. of the 2011 ACM SIGCOMM Conference on Internet Measurement Conference, ser. IMC 11. New York, NY, USA: ACM, pp. 137-154, 2011. Article (CrossRef Link)

[25] D. Croce, D. Garlisi, F. Giuliano, and I. Tinnirello, "Learning from errors: Detecting zigbee interference in wifi networks," in Proc. of 2014 13th Annual Mediterranean Ad Hoc Networking Workshop, pp. 158-163, June. 2014. Article (CrossRef Link)

[26] Y. Zhang and Q. Li, "Howies: A holistic approach to zigbee assisted wifi energy savings in mobile devices," in Proc. of 2013 Proceedings IEEE INFOCOM, pp. 1366-1374, April. 2013. Article (CrossRef Link)

[27] Z. Qin, Y. Sun, J. Hu, W. Zhou, and J. Liu, "Enhancing efficient link performance in zigbee under cross-technology interference,” Mobile Networks and Applications, vol. 25, pp. 68-81, 2020. Article (CrossRef Link)

[28] R. Chen and W. Gao, "Enabling cross-technology coexistence for extremely weak wireless devices," in Proc. of IEEE INFOCOM 2019 - IEEE Conference on Computer Communications, 2019. Article (CrossRef Link)

[29] Y. Chae and S. M. Kim, "Safeguarded zigbee via wifi guard band," in Proc. of the 16th ACM Conference on Embedded Networked Sensor Systems, pp. 355-356, 2018. Article (CrossRef Link) 
[30] G. Chen, W. Dong, Z. Zhao, and T. Gu, "Accurate corruption estimation in zigbee under cross-technology interference,” IEEE Transactions on Mobile Computing, vol. 18, pp. 2243-2256, 2019. Article (CrossRef Link)

[31] S. M. Kim and T. He, "Freebee: Cross-technology communication via free side-channel," in Proc. of the 21st Annual International Conference on Mobile Computing and Networking, New York, USA: ACM, pp. 317-330, 2015. Article (CrossRef Link)

[32] T. Mangir, L. Sarakbi, and H. Younan, "Analyzing the impact of wi-fi interference on zigbee networks based on real time experiments,” 2011.

[33] R. M. Marcacini, J. C. Carnevali, and J. Domingos, “On combining websensors and dtw distance for knn time series forecasting," in Proc. of 2016 23rd International Conference on Pattern Recognition, pp. 2521-2525, Dec. 2016. Article (CrossRef Link)

[34] H. Abdi, L. J. Williams, and D. Valentin, "Multiple factor analysis: Principal component analysis for multitable and multiblock data sets,” WIREs Comput. Stat., vol. 5, no. 2, pp. 149-179, Mar. 2013. Article (CrossRef Link)

[35] D. Etter and C. Domeniconi, “Multi2Rank: Multimedia Multiview Ranking,” in Proc. of 2015 IEEE International Conference on Multimedia Big Data, Beijing, pp. 80-87, 2015.

Article (CrossRef Link) 


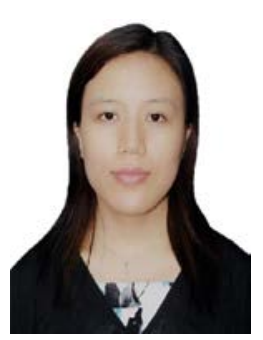

Dian Zhang received the PhD degree in computer science and engineering from the Hong Kong University of Science and Technology(HKUST), Hong Kong, in 2010. After that, she worked as a research assistant professor at the Fok Ying Tung Graduate School, HKUST. From 2012 to 2019, she worked as an associate professor at Shenzhen university. She is currently an associate professor at Lingnan University, Hong Kong. Her research interests include big data analytics and mobile computing. She is a member of the IEEE.

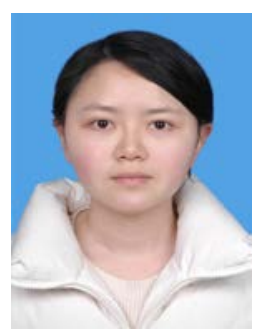

Rujun Zhang is currently a Master student in the College of Computer Science and Software Engineering, Shenzhen University. Her research interests focus on wireless sensor networks.

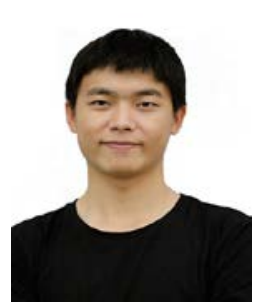

Haizhou Guo is currently a postgraduate student in the College of Computer Science and Software Engineering, Shenzhen University. His research interests include machine learning, neural networks and spatiotemporal data mining.

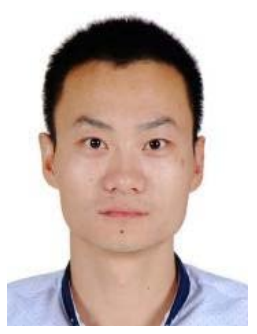

Peng Xiang received the Master's degree in software engineering from the Shenzhen University, China, in 2017.He is currently a Engineer in the Shenzhen Hai Yun Tian science and technology incorporated company, China. His research interests focus on wireless sensor networks.

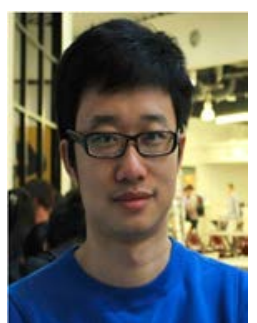

Xiaonan Guo received the PhD degree in computer science and engineering from the Hong Kong university of Science and Technology, Hong Kong. He is currently a Postdoctoral Research in the Data Analysis and Information Security Lab of the Department of Electrical and Computer Engineering at Stevens Institute of Technology. His research interests include Cyber Security and Privacy, Security in Mobile Devices, IoT security, Intrusion Detection Using WiFi for Smart Homes, Mobile Healthcare and Large Data Analysis on Mobile Devices. He is a member of the IEEE. 